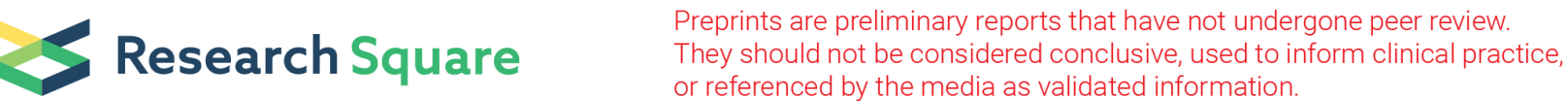

\section{Mechanical Growth Factor Promotes FOXP3 \\ Expression in Regulatory T Cells and Enhances Its Function in Treating Ankylosing Spondylitis}

\section{Hongyu Qin}

The First Affiliated Hospital of Guangxi Medical University

\section{Shuangshuang Yuan}

The First Affiliated Hospital of Guangxi Medical University

Hao Zeng

The First Affiliated Hospital of Guangxi Medical University

Hao Li

The First Affiliated Hospital of Guangxi Medical University

Jinsong Yang ( $\sim$ JinsongYangGxmu@163.com )

The First Affiliated Hospital of Guangxi Medical University

\section{Research Article}

Keywords: ankylosing spondylitis, FOXP3, mechanical growth factor, rat model, regulatory T cell, treatment

Posted Date: May 17th, 2021

DOI: https://doi.org/10.21203/rs.3.rs-238795/v2

License: (c) (1) This work is licensed under a Creative Commons Attribution 4.0 International License. Read Full License 


\section{Abstract}

Objective: We aimed to verify whether mechanical growth factor (MGF) may be an effective target for treating ankylosing spondylitis.

Methods: FOXP3 expression was measured in Treg cells from healthy male subjects administered MGF. A rat model of ankylosing spondylitis was established, and the level of ankylosing spondylitis-related factors (tumor necrosis factor [TNF]-a, interleukin [IL]-2, and IL-10) was measured.

Results: We found that the proliferation and total number of Treg cells, as well as FOXP3 expression, significantly increased in the MGF-treated groups compared with those in the control. The level of inflammation, bone destruction, and new bone formation significantly decreased in rats treated with MGF compared with those in the control group. TNF-a expression significantly decreased, whereas the IL-2 and IL-10 levels significantly increased in the MGF group compared with those in the control.

Conclusions: MGF may delay disease progression in ankylosing rats by inducing FOXP3 expression, promoting FOXP3+ Treg cell proliferation and differentiation, reducing TNF-a expression, and increasing IL-10 and IL-2 expression.

\section{Introduction}

Ankylosing spondylitis (AS) is an autoimmune disease that causes inflammation of the sacroiliac joints and muscle attachment points and is closely related to HLA-B27. It typically begins to develop around the age of 30 years, affecting the central axis; inflammation of the sacroiliac joint is an important feature of this disease ${ }^{[1]}$. As the disease progresses, calcification and rigidity of the annulus fibrosus and nearby ligaments often occur. The clinical manifestations of AS include lower back pain and reduced spine mobility, which frequently lead to long-term patient disability and cause serious psychological and physiological damage. Non-steroidal anti-inflammatory drugs remain the first-line recommended treatment for $\mathrm{AS}^{[2]}$. Conventional synthetic disease-modifying antirheumatic drugs such as methotrexate and sulfadiazine may slightly improve peripheral symptoms but are ineffective for treating axial manifestations ${ }^{[3]}$. For patients who fail to respond to non-steroidal anti-inflammatory drugs, biological disease anti-rheumatic drugs, mainly tumor necrosis factor (TNF) inhibitors, are among the most powerful treatment options ${ }^{[2]}$. However, TNF-a antagonists are only effective in $40 \%$ of patients, and their associated high risks of infection and tumor development ${ }^{[4]}$ have prompted researchers to identify safer and more effective treatments. Among them, regulatory $\mathrm{T}$ (Treg) cells have garnered increasing attention $^{[5]}$.

Regulatory $\mathrm{T}$ lymphocytes comprise a small subset of $\mathrm{CD} 4+\mathrm{T}$ cells and play crucial roles in maintaining immune tolerance and preventing autoimmunity ${ }^{[6]}$. In 1995, Sakaguchi first identified Treg cells as a subset of CD4+ T cells with constitutive CD25 expression ${ }^{[7]}$. In 2003, the forkhead box P3 (FOXP3) transcription factor was identified as a key transcription factor of Treg cells and was later identified as an 
essential component for the development, maturation, and immunosuppressive functions of Treg cells ${ }^{[8]}$. The CD4, CD25, and CD127 antibodies can be labeled to effectively isolate CD4+CD25+FOXP3+ Treg cells ${ }^{[9,10]}$. A previous study showed that Treg cells from patients with AS have functional defects and play important roles in $A S^{[5]}$. Therefore, it is important to determine how the proliferation and immunetolerance functions of Treg cells can be enhanced in AS. Enhancement of Treg cell functions has emerged as a promising strategy and new direction for treating AS.

AS mostly affects the central axis of the spine, sacroiliac joint, and Achilles tendon, which are subjected to the highest mechanical loads in the body. Additionally, ossification starts at the attachment points of muscles, which also experience concentrated mechanical loads. Thus, there is a relationship between mechanical load and AS. In terms of disease treatment, functional exercise can effectively alleviate the symptoms of AS in the affected patients ${ }^{[11]}$. The painful symptoms in patients with AS are aggravated at night and patients cannot remain in bed for a long time. However, if patients get up and move their waist, the symptoms are rapidly relieved. Additionally, if a patient engages in sufficient physical activity during the day, the pain at night is reduced or it even disappears, and the sleep quality of patients is substantially improved. Karatay et al ${ }^{[12]}$ found that functional exercise can effectively increase the expression of insulin-like growth factor-1 (IGF-1) in the peripheral blood, suggesting that IGF1 can relieve disease symptoms in patients with AS.

Mechanical growth factor (MGF, also known as mechano growth factor or force growth factor) is an alternative splicing variant of IGF-1. All mRNA splice variants of IGF-1 contain exons 3 and 4 . Direct splicing of mRNA exons 4 and 6 results in the formation of IGF-1 Ea, whereas continuous splicing of exons 4-6 generates MGF, which is referred to as IGF-1 Ec in humans and IGF-1 Eb in rodents ${ }^{[13]}$. Previous studies have confirmed that mechanical loading and muscle damage can induce MGF expression and that MGF is sensitive to mechanical stimulation ${ }^{[14]}$. Interestingly, IGF-1 can specifically promote Treg cell proliferation and differentiation and elicit a therapeutic effect by enhancing immune tolerance in various animal models of immune diseases ${ }^{[15]}$. Therefore, we hypothesized that MGF can enhance or restore the body's immune tolerance and immune suppression functions by increasing the number of Treg cells and their functionality, thereby improving their therapeutic effects against AS. In the present study, we used a rat model of AS to explore the effects of MGF in AS in order to establish whether a treatment strategy targeting MGF and Treg cells is effective for AS.

\section{Materials And Methods}

\subsection{Flow-cytometric sorting of Treg cells}

Peripheral venous blood samples were obtained from six healthy male college students aged 23-27 years in the First Affiliated Hospital of Guangxi Medical University. Evaluations were performed to exclude systemic lupus erythematosus, rheumatoid arthritis, systemic vasculitis, scleroderma, pemphigus, dermatomyositis, mixed connective-tissue disease, ulcerative colitis, and other autoimmune diseases. The study was approved by the Institutional Ethics Committee of the First Affiliated Hospital of Guangxi 
Medical University $₫ \mathrm{KY}-\mathrm{E}-141 \rrbracket$, and all subjects signed an informed consent form. Fresh peripheral venous blood was drawn from volunteers and stored in a heparin-anticoagulation tube at $4^{\circ} \mathrm{C}$. Next, $4 \mathrm{~mL}$ of lymphocyte separation solution (Stemcell Technologies, Vancouver, Canada) was added to a $15-\mathrm{mL}$ centrifuge tube, and then slowly added $8 \mathrm{~mL}$ of blood at $1 \mathrm{~cm}$ above the liquid surface of the centrifuge tube to avoid the liquids from mixing with each other. The tube was centrifuged at $2000 \mathrm{rpm}$ for $30 \mathrm{~min}$ in a low-speed centrifuge at $25^{\circ} \mathrm{C}$. After centrifugation, the cells in the centrifuge tube were divided into four layers from top to bottom. The first layer was blood plasma, second layer was ring-shaped milky white lymphocytes, third layer was transparent separation liquid, and fourth layer was red blood cells. CD4+CD25+CD127- Treg cells (CD4+CD25+) were isolated from the second layer. Briefly, a CD127 MicroBead Kit (Miltenyi Biotec, Gladbach Bergisch, Germany) was used to isolate CD4+CD127- cells, and a CytoFLEX flow cytometer (Beckman Coulter, Brea, CA, USA) was used to sort CD4+CD25+ Treg cells. To determine the purity of the isolated Treg cells, we used a fluorescein isothiocyanate (FITC)-conjugated CD4 antibody, allophycocyanin-conjugated CD25 antibody, phycoerythrin-conjugated CD127 antibody, phycoerythrin-conjugated rat IgG2K isotype control, allophycocyanin-conjugated rat IgG1K isotype control, and FITC-conjugated rat IgG2K isotype control (eBioscience, San Diego, CA, USA). All experiments were performed in accordance with relevant guidelines and regulations (Supplemental data).

\subsection{Expansion of Treg cells in culture}

Treg cell expansion medium, containing $500 \mathrm{lU} / \mathrm{mL}$ Human IL2 Improved Sequence, premium grade (Miltenyi Biotec), 5\% human AB serum (GEMINI, West Sacramento, CA, USA), $0.01 \mathrm{mM} \beta$-mercaptoethanol (Solebold, Beijing, China), $1 \%$ penicillin (Solebold), and $100 \mathrm{nmol} / \mathrm{L}$ rapamycin (Solebold), was prepared, and the mixture was added to $500 \mathrm{~mL}$ of TexMACS GMP Medium (Miltenyi Biotec). The extracted Treg cells were resuspended in the expansion medium at a concentration of $1 \times 106 \mathrm{cells} / \mathrm{mL}$. Aliquots of the cell suspension were added to U-shaped 96 -well plates $(100 \mu \mathrm{L}$ per well), followed by the addition of antiCD3/CD28 MACSiBead ${ }^{\text {TM }}$ particles (Miltenyi Biotec). The 96 -well plates were incubated at $37^{\circ} \mathrm{C}$ in $5 \%$ carbon dioxide for 7 days. During the incubation period, the spent medium was removed, and fresh medium was added based on the color of the culture medium and morphology of the cells under a microscope. After 7 days, the expansion was complete, and magnetic beads were removed using a MACSiMAG Separator (Miltenyi Biotec).

\subsection{Carboxyfluorescein succinimidyl ester (CFSE) labeling of Treg cells}

Sorted CD4+CD25+CD127- Treg cells were divided into two groups. One group was fixed with $2 \%$ paraformaldehyde and regarded as negative cells. The other group was stained with CFSE (eBioscience) and fixed in $2 \%$ paraformaldehyde, after which the fluorescence intensity of the two groups was measured and compared.

\subsection{Co-culture of Treg cells and MGF}

CFSE-stained Treg cells were divided into five groups, each of which was sub-divided into three fractions. The five groups were the control group, 100 nM MGF group, 200 nM MGF group, 400 nM MGF group, and 
$800 \mathrm{nM}$ MGF group. Each group was mixed with the same amount of Treg cell suspension and antiCD3/CD28 MACSiBead ${ }^{\text {TM }}$ particles (Miltenyi Biotec) and saline or 100, 200, 400, or 800 nM MGF (Kangtide Biotechnology, Beijing, China). The Treg cells were cultured for 7 days, the magnetic beads were removed, and cell proliferation was analyzed by measuring fluorescence in the FITC channel by flow cytometry.

\subsection{Effect of MGF on FOXP3 expression in Treg cells}

Based on the results obtained after co-culturing the Treg cells with MGF, we co-cultured Treg cells in normal saline or 800 nM MGF. RNA extraction, reverse transcription, and FOXP3 cDNA amplification from both groups of Treg cells were performed using a micro RNA extraction kit, high-efficiency reverse transcription kit, and fluorescent quantitative PCR kit (eBioscience), respectively. FOXP3 cDNA was amplified using the forward primer 5'-CTCTTCTTCCTTGAACCCCAT3' and reverse primer 5'CTGGAGGAGTGCCTGTAAG-3', which were obtained from Shanghai Shenggong Biological Company (Shanghai, China).

\subsection{Animals}

We obtained 6-12-week-old male and female HLA-B27/Huß2m transgenic rats weighing 250-300 g from the Rheumatology Team of the University of Texas Southwestern Medical Center (Dallas, TX, USA) ${ }^{[16]}$. This rat strain possesses 20 copies of HLA-B27 and 50 copies of the Hu- $\beta 2 m$ microglobulin gene ${ }^{[16-18]}$ and is the most representative available animal model of AS. All animal breeding and experiments were carried out in a specific pathogen-free laboratory of the Animal Experiment Center of Guangxi Medical University. The study was approved by the Institutional Ethics Committeethe of the First Affiliated Hospital of Guangxi Medical University and was carried out in compliance with the ARRIVE guidelines. \KY-E-141凶.

\subsection{Orchiectomy}

Under normal circumstances, male HLA-B27/Huß2m transgenic rats have an $80 \%$ incidence rate of ankle arthritis between days 100 and 200 and a 40\% incidence rate of spondylitis between days 125 and 225 . In contrast, female B27/Huß2m transgenic rats do not develop the disease. The reason for this difference is that epididymo-orchitis is crucial for the pathogenesis of subsequent SA in male rats ${ }^{[17]}$. Therefore, to avoid interference, we performed bilateral epididymal testis castration on 4-week-old male HLA$\mathrm{B} 27 / \mathrm{Hu} 32 \mathrm{~m}$ transgenic rats based on a standard method ${ }^{[19]}$.

\subsection{Immunization}

To increase the incidence of AS and shorten the test period, $90 \mu \mathrm{g}$ of inactivated Mycobacterium tuberculosis (Solarbio, Beijing, China), dissolved in incomplete Freund's adjuvant, was injected subcutaneously at the base of the tail of 6-week-old female and castrated male rats. This was performed to increase the incidence of rat SA to $100 \%$ and substantially shorten the time of onset ${ }^{[18]}$. 


\subsection{Preventive treatment with MGF}

Eighteen HLA-B27/Huß2m transgenic rats were immunized with $\mathrm{M}$. tuberculosis and randomly divided into a control group, low-dose MGF group, or high-dose MGF group ( $\mathrm{n}=6$ rats/group). Preventive treatment with MGF was started 1 week after M. tuberculosis immunization. The control group was intraperitoneally injected with $3 \mathrm{~mL} / \mathrm{kg}$ normal saline, low-dose MGF group was intraperitoneally injected with $3 \mu \mathrm{g} \mathrm{MGF} / \mathrm{kg}$, and the high-dose MGF group was intraperitoneally injected with $10 \mu \mathrm{g} \mathrm{MGF/kg}$ every 3 days. MGF preventive treatment lasted 7 weeks, when the ankles and tail vertebrae of rats were photographed and recorded to measure the severity of symptoms.

\subsection{Serological analysis}

Rats were sacrificed by cervical dislocation. Rat ankles and spine joints were cut into bone fragments ( $\leq 1$ $\mathrm{cm}$ ) and placed in ethylenediaminetetraacetic acid decalcified solution (Sangon, Shanghai, China). The ratio of the solution to the volume of bone tissue was greater than 20 . The bone fragments were incubated in ethylenediaminetetraacetic acid decalcification solution at room temperature, and the solution was replaced every 3 days. Decalcification was considered as complete when a syringe needle could easily penetrate the bone compact without resistance. Bone sections were stained with hematoxylin and eosin (H\&E) using standard methods to observe inflammation and bone destruction, and the sections were stained with safranin $\mathrm{O}$ to observe new bone formation.

\subsection{Histological analysis}

Rats were sacrificed by cervical dislocation. Rat ankles and spine joints were cut into bone fragments ( $\leq 1$ $\mathrm{cm}$ ) and placed in ethylenediaminetetraacetic acid (EDTA) decalcified solution (Sangon, Shanghai). The ratio of the solution to the volume of bone tissue was greater than 20 . The bone fragments were incubated in the EDTA decalcification solution at room temperature, and the solution was replaced every 3 days. Decalcification was considered to be complete when a syringe needle could easily penetrate the bone compact without resistance. Bone sections were stained with H\&E according to standard methods to observe inflammation and bone destruction, and sections were stained with safranin 0 to observe new bone formation.

\subsection{Histological score}

Each joint sample was treated as an independent sample for histological analysis. Two independent observers (QHY and YSS) semi-quantitatively scored the stained samples. According to similar research, (20) the score was based on three factors: inflammation, bone destruction, and new bone formation. Each parameter was assigned a score ranging from 0 to $3(0=$ normal, $1=$ mild influence, $2=$ moderate influence, 3 = severe influence), and the average sum of 10 fields under a microscope was determined to be the final joint score of each sample.

\subsection{Statistical analysis}


FlowJo software (version 10; TreeStar, Ashland, OR, USA) was used to graph the cell proliferation data obtained by flow cytometry. GraphPad software (version 9.0, GraphPad, Inc., La Jolla, CA, USA) and SPSS Statistics software (version 24, SPSS, Inc., Chicago, IL, USA) were used for statistical analyses. Differences between groups were compared by independent sample t-test, and differences among multiple groups were compared using the one-way analysis of variance. $P<0.05$ was used as the threshold for statistically significant differences $\left({ }^{\star} P<0.05 ; * \star P<0.01 ; * \star * P<0.001\right)$.

\section{Results}

\subsection{Effect of MGF on in vitro proliferation of and FOXP3 expression in human peripheral blood CD4+CD25+CD127-Treg cells}

After sorting, the purity of CD4+CD25+CD127-Treg cells was $91.04 \%$, which was sufficient for the subsequent experiments (Supplementary Fig. 1). CFSE staining was performed to determine the percentage of Treg cells in each group relative to the total number of cells. The average percentage of Treg cells in the control group was $48.63 \% \pm 0.12 \%$ (Fig. 1A), whereas that in the 100, 200, 400, and 800 nm MGF groups was $49.97 \% \pm 0.47 \%$ (Fig. 1B), $52.40 \% \pm 0.93 \%$ (Fig. 1C), 58.67\% $\pm 0.83 \%$ (Fig. 1D), and $67.73 \% \pm 2.29 \%$ (Fig. 1E), respectively. As the MGF concentration increased, the proportion of proliferating cells increased. The percentage of proliferating cells (relative to the total number of cells) increased significantly $(P<0.01)$ in the 400 and 800 nM MGF groups compared with that in the control group (Fig. 1F). The effect of MGF treatment on Treg cell proliferation was measured by comparing FOXP3 expression levels in the control and experimental (800 nM MGF) groups. FOXP3 expression in the MGF experimental group significantly increased to $1.47 \pm 0.12$-fold compared with that in the control group (Fig. 1G), indicating that MGF increased the number and activity of Treg cells.

\subsection{Effect of MGF on the clinical symptoms in HLA-B27/Huß2m transgenic rats and differences in the serum IL-10, IL-2, and TNF- $a$ levels in rats before and after MGF treatment}

After immunizing rats in different groups with inactivated $\mathrm{M}$. tuberculosis, we scored the degree of inflammation in the wrists and arthritis in the ankles and spinal joints. After 7 weeks of prophylactic MGF treatment, the symptom severity of the wrists, ankles, and spinal joints significantly differed in each group. Swelling of the front paw, back grasping joints, and spine joints was the most severe in the control group (Fig. 2A-C) and was the lowest in the high-dose MGF group (Fig. 2G-I), followed by the low-dose MGF group (Fig. 2D-F).

Additionally, the skin around the wrists and ankle joints in the high-dose MGF group had a normal color and the tail structure was normal, whereas the skin around the spine in the low-dose MGF and control groups showed persistent erythema and bamboo-like morphology. The arthritis score in the high-dose MGF group was significantly lower $(P<0.05)$ than that in the control group (Fig. $3 A)$. Our results show that intraperitoneal injection of high-dose MGF reduced the severity of SA in rats. 
Before MGF injection, the serum levels of IL-2, IL-10, and TNF-a in rats in each group did not significantly differ. After 7 weeks of MGF intervention, the serum IL-2 level in rats in the high-dose MGF group (1117.95 $\pm 9.86 \mathrm{pg} / \mathrm{mL}$ ) was higher than that in the control group $(952.50 \pm 30.29 \mathrm{pg} / \mathrm{mL} ; \mathrm{P}<0.01 ;$ Fig. 3B). The serum IL-10 level in the high-dose MGF $(9.02 \pm 0.85 \mathrm{pg} / \mathrm{mL})$ and low-dose MGF $(8.53 \pm 0.83 \mathrm{pg} / \mathrm{mL})$ groups was higher than that in the control group $(5.27 \pm 1.03 \mathrm{pg} / \mathrm{mL} ; \mathrm{P}<0.05 ;$ Fig. $3 \mathrm{C})$. The serum level of TNG-a in the high-dose MGF group (15.54 $\pm 0.09 \mathrm{pg} / \mathrm{mL})$ and low-dose MGF $(18.04 \pm 1.80 \mathrm{pg} / \mathrm{mL})$ groups was significantly lower than that in the control group $(21.30 \pm 2.32 \mathrm{pg} / \mathrm{mL} ; \mathrm{P}<0.01$; Fig. 3D).

$\mathrm{H} \& \mathrm{E}$ and safranin $\mathrm{O}$ staining of the rat spinal joint sections showed that inflammation, the number of new bone-forming cells, and bone destruction significantly decreased in a dose-dependent manner in the MGFtreated groups compared with those in the control group (Fig. 4).

\section{Discussion}

Ankylosing spondylitis is an autoimmune disease involving inflammation of the joints and muscle attachment points. As the disease progresses, the annulus fibrosus and surrounding ligaments gradually become inflamed and calcified, leading to progressive stiffness, bone destruction, and new bone formation. Studies on autoimmune diseases have mainly focused on Treg cells and Th17 cells ${ }^{[20]}$. Importantly, Treg cells are important for immune tolerance and immune system maintenance. Defects in Treg cell function play a vital role in the pathogenesis of autoimmune diseases ${ }^{[21]}$. It has been confirmed that the percentage of Treg cells in the blood of patients with certain autoimmune diseases may decrease, increase, or remain unchanged. However, it is considered that Treg cells function abnormally in patients with autoimmune diseases. The number of Treg cells in the peripheral blood circulation or target organs of patients with autoimmune diseases is normal, but their immune suppression and immune tolerance functions are reduced ${ }^{[22]}$. CD4+CD25+ Treg cells expressing FOXP3 maintain immune regulation and immune tolerance by removing abnormal substances harmful to the host or preventing excessive immune system responses to maintain immunity ${ }^{[23]}$. FOXP3 plays a key role in the development and function of Treg cells ${ }^{[24]}$. Mutations in human FOXP3 can lead to incomplete development of Treg cells and affect their normal immune tolerance and immune regulation functions. This in turn can promote the development of various immune diseases, including AS, inflammatory bowel disease, psoriatic arthritis, systemic lupus erythematosus, and dry synthesis by allowing Treg cells to perform their normal functions. Here, we evaluated the effect of MGF on the proliferation of human CD4+CD25+CD127-Treg cells in vitro. Additionally, the expression level of FOXP3 in Treg cells treated with MGF significantly exceeded the above. This indicates that MGF can promote the proliferation and function of Treg cells. Previous studies have shown that IGF-1 can directly promote the proliferation of Treg cells, enhance their immunosuppressive function, and exert a protective effect against autoimmune diseases, and IGF-1 has no effect on TH0, TH1, and TH17 cells ${ }^{[15]}$. MGF is used as an IGF-1 variant, while enhancing the function of Treg cells; whether it has an effect on other immune cells still needs further research. 
Previous studies have reported that $100-1,000 \mu \mathrm{g}$ of heat-inactivated M. tuberculosis can only induce artificial organs in wild-type rats ${ }^{[25-28]}$. In 1961 , it was reported that immunization with a high dose of heat-inactivated M. tuberculosis (five times with $500 \mu \mathrm{g}$ ) could cause spondylitis in wild-type rats ${ }^{[29]}$ and this requires consideration in terms of safety and ethics. We used the HLA-B27 transgene and established methods for suture, bone destruction, and new bone formation of rat spinal joints after MGF treatment. The severity of histopathological manifestations was reduced by MGF treatment. The rats in the highdose MGF group did not produce new bone tissue, and local resection and infiltration were significantly improved. This suggests that MGF can relieve the symptoms of AS.

To determine the molecular mechanism by which MGF exerts an obvious protective effect against AS, we measured the levels of the cell death markers TNF- $a$, IL-2, and IL-10 in the serum of AS model rats. TNF- $a$ is a pro-inflammatory cytokine mainly produced by inflammatory cells such as monocytes, macrophages, and activated T cells. TNF-a enhances the inflammatory response of AS, intervertebral disc degeneration ${ }^{[30]}$ and thrombosis ${ }^{[31,32]}$. Our experiments showed that before MGF intervention, there was no significant difference in the serum TNF-a level of rats in each group. After MGF treatment, MGF reduced the serum TNF-a levels in AS rat, leading to reduced symptoms of AS.

IL-10 is a pleiotropic anti-inflammatory and immunosuppressive cytokine. An imbalance in the IL-10 level plays an important role in the development of autoimmune diseases such as rheumatoid arthritis, Crohn's disease, and multiple sclerosis ${ }^{[33]}$.In the presence of proinflammatory signals, almost all immune cells (including cytotoxic T lymphocytes, CD4+ T cells, NK cells, B cells, and macrophages) produce IL-10 to exert anti-inflammatory effects. Recent studies have showed that IL-10 can enhance the function of Treg and may be useful for treating autoimmune diseases ${ }^{[34]}$. In our study, before MGF intervention, the serum IL-10 level in rats in each group was not significantly different; after MGF treatment, the IL-10 level significantly increased in the MGF group, and it was the lowest in the control group. This indicates that MGF can promote the immune cells in AS rats to produce more IL-10 to inhibit excessive inflammation and relieve the symptoms of AS.

IL-2 is one of the most widely studied cytokines and has a wide range of functions, especially in the immune system, which plays an important role in maintaining immune homeostasis ${ }^{[34]}$. IL-2 is mainly secreted by activated CD4+ T cells ${ }^{[35]}$. In our experiment, after MGF treatment, the level of IL-2 in the highdose MGF group was significantly higher than that in the normal saline control group, and the difference was significant. MGF might activate CD4+ T cells, so that they can secrete more IL-2 and increase serum IL-2 level. IL-2 promotes the proliferation of CD4+CD25+ Treg cells; it is a key cytokine for immune tolerance ${ }^{[35-37]}$. Studies have found that the surface of Treg cells has a large number of interleukin 2 receptors (IL-2R). After binding to IL-2R on T cells, IL-2 activates the JAK/STAT signal transduction pathway of T cells, leading to the phosphorylation of the STAT 5 transcription factor. STAT 5 combines with the Foxp3 promoter to induce Foxp3 expression, promote the proliferation and differentiation of Treg cells, and enhance its function ${ }^{[36]}$. This suggests that MGF likely increases the level of IL-2 to promote Foxp3 expression, thereby promoting the proliferation of Treg cells and enhancing their functions. 


\section{Limitations}

Previous studies have shown that IGF-1 can directly promote the proliferation of Treg cells, enhance their immunosuppressive function, and have a protective effect on autoimmune diseases.MGF is used as IGF -1 variants, while enhancing the function of Treg cells, whether it has an effect on other immune cells still needs further research.

\section{Conclusions}

In conclusion, our results suggest that MGF likely induces FOXP3 expression by increasing serum IL-2 level, promoting Treg cell proliferation and differentiation, and enhancing their functions. Furthermore, MGF upregulates the secretion of IL-10 (an anti-inflammatory factor) and downregulates the secretion of TNF-a (a pro-inflammatory factor) in Treg cells, thereby effectively alleviating the symptoms of AS in rats. These findings suggest that MGF can be used as a new strategy for AS treatment.

\section{Abbreviations}

AS, ankylosing spondylitis; FOXP3, forkhead box P3; H\&E, hematoxylin and eosin; IGF-1, insulin-like growth factor-1; IL, interleukin; MGF, mechanical growth factor; PBMC, peripheral blood mononuclear cell; TNF, tumor necrosis factor; Treg, regulatory $T$ cell

\section{Declarations}

\section{Acknowledgements}

We thank our colleagues from the Animal Experiment Center of Guangxi Medical University for their support and collaboration.

\section{Author contributions}

YJS designed and supervised the overall research. QHY and YSS conducted rat feeding and rat experiment. LH conducted human peripheral blood collection and analysis. ZH and QHY provided pathology assistance and performed image editing. QHY and YJS wrote and revised the manuscript. All authors read and approved the manuscript.

\section{Conflicts of interest}

The authors have no conflicts of interest to disclose in relation to this article.

\section{Availability of data and materials}

All data needed to achieve the conclusion are presented in the paper. 
Ethics approval was obtained for the study (KY-E-141). Informed consent was obtained from the patient to participate in the study.

\section{Consent for publication}

Informed written consent was obtained from the patient for publication of this case report and accompanying images.

\section{Funding}

This work was supported by the Guangxi Natural Science Foundation (No. 2017GXNSFAA198127). The funding agency funded the collection and analysis of samples in this study. It had no role in the collection, analysis, and interpretation of data, in the writing of the report, or in the decision to submit the article for publication.

\section{References}

[1] Garcia-Montoya L, Gul H, Emery P. Recent advances in ankylosing spondylitis: understanding the disease and management. F1000Research. 7,1512 (2018)

[2] Ward MM, Deodhar A, Gensler LS, Dubreuil M. 2019 Update of the American College of Rheumatology/Spondylitis Association of America/Spondyloarthritis Research and Treatment Network Recommendations for the Treatment of Ankylosing Spondylitis and Nonradiographic .Axial Spondyloarthritis. 71,1599-613. (2019)

[3] Haibel H, Brandt HC, Song IH, Brandt A, Listing J, Rudwaleit M, et al. No efficacy of subcutaneous methotrexate in active ankylosing spondylitis: a 16-week open-label trial. Annals of the rheumatic diseases.66,419-21 (2007)

[4] Ma Z, Liu X, Xu X, Jiang J, Zhou J, Wang J, et al. Safety of tumor necrosis factor-alpha inhibitors for treatment of ankylosing spondylitis. Medicine.96,e7145(2017)

[5] Zhu W, He X, Cheng K, Zhang L, Chen D, Wang X, et al. Ankylosing spondylitis: etiology, pathogenesis, and treatments. Bone research.7, 22 (2019)

[6] Nie J, Li YY, Zheng SG, Tsun A, Li B. FOXP3(+) Treg Cells and Gender Bias in Autoimmune Diseases. Frontiers in immunology. 6,493 (2015)

[7] Sakaguchi S, Sakaguchi N, Asano M, Itoh M, Toda M. Immunologic self-tolerance maintained by activated T cells expressing IL-2 receptor alpha-chains (CD25).

Breakdown of a single mechanism of self-tolerance causes various autoimmune diseases. Journal of immunology (Baltimore, Md : 1950). 155,1151-64(1995) 
[8] Fontenot JD, Gavin MA, Rudensky AY. Foxp3 programs the development and function of CD4+CD25+ regulatory T cells. Nature immunology. 4,330-6(2003)

[9] Kmieciak M, Gowda M, Graham L, Godder K, Bear HD, Marincola FM, et al. Human T cells express CD25 and Foxp3 upon activation and exhibit effector/memory phenotypes without any regulatory/suppressor function. Journal of Translational Medicine. 7,89 (2009)

[10] Liu W, Putnam AL, Xu-yu Z, Szot GL, Lee MR, Zhu S, et al. CD127 expression inversely correlates with FoxP3 and suppressive function of human CD4+ T reg cells. Journal of Experimental Medicine. 203,170111(2006)

[11] Analay Y, Ozcan E, Karan A, Diracoglu D, Aydin R. The effectiveness of intensive group exercise on patients with ankylosing spondylitis. Clinical rehabilitation. 17,631-6(2003)

[12] Karatay S, Yildirim K, Melikoglu MA, Akcay F, Şenel K. Effects of dynamic exercise on circulating IGF1 and IGFBP-3 levels in patients with rheumatoid arthritis or ankylosing spondylitis. Clinical rheumatology.26,1635-9(2007)

[13] Matheny RW, Jr., Nindl BC, Adamo ML. Minireview: Mechano-growth factor: a putative product of IGFI gene expression involved in tissue repair and regeneration. Endocrinology. 151,865-75(2010)

[14] Yang SY, Goldspink G. Different roles of the IGF-I Ec peptide (MGF) and mature IGF-I in myoblast proliferation and differentiation. FEBS letters. 522,156-60(2002)

[15] Bilbao D, Luciani L, Johannesson B, Piszczek A, Rosenthal N. Insulin-like growth factor-1 stimulates regulatory T cells and suppresses autoimmune disease. EMBO Molecular Medicine.6,1423-35(2014)

[16] Tran TM, Dorris ML, Satumtira N, Richardson JA, Hammer RE, Shang J, et al. Additional human beta2-microglobulin curbs HLA-B27 misfolding and promotes arthritis and spondylitis without colitis in male HLA-B27-transgenic rats. Arthritis and rheumatism. 54,1317-27(2006)

[17] Taurog JD, Rival C, van Duivenvoorde LM, Satumtira N, Dorris ML, Sun M, et al. Autoimmune epididymoorchitis is essential to the pathogenesis of male-specific spondylarthritis in HLA-B27transgenic rats. Arthritis and rheumatism.

\section{8,2518-28(2012)}

[18] van Tok MN, Satumtira N, Dorris M, Pots D, Slobodin G, van de Sande MG, et al. Innate Immune Activation Can Trigger Experimental Spondyloarthritis in HLA-B27/Hubeta2m Transgenic Rats. Frontiers in immunology. 8,920(2017)

[19] Waynforth H, Flecknell P. Experimental and Surgical Technique in the Rat. 2. London: Academic Press. 275-276(1992) 
[20] van Duivenvoorde LM, Dorris ML, Satumtira N, van Tok MN, Redlich K, Tak PP, et al. Relationship between inflammation, bone destruction, and osteoproliferation in the HLA-B27/human $\beta 2$ microglobulin-transgenic rat model of spondylarthritis. Arthritis and rheumatism.10,3210-9(2012)

[21] Benedek TG. How did ankylosing spondylitis become a separate disease? Clinical and experimental rheumatology.4,S3-9(2009)

[22] Brown MA, Laval SH, Brophy S, Calin A. Recurrence risk modelling of the genetic susceptibility to ankylosing spondylitis. Annals of the rheumatic diseases.11,883-6(2000)

[23] Bobacz K, Ullrich R, Amoyo L, Erlacher L, Smolen JS, Graninger WB. Stimulatory effects of distinct members of the bone morphogenetic protein family on ligament fibroblasts. Annals of the rheumatic diseases.2,169-77(2006)

[24] Inanc B, Elcin AE, Elcin YM. Osteogenic induction of human periodontal ligament fibroblasts under two- and three-dimensional culture conditions. Tissue engineering. 12,257-66(2006)

[25] Waksman BH, Pearson CM, Sharp JT. Studies of arthritis and other lesions induced in rats by injection of mycobacterial adjuvant. II. Evidence that the disease is a disseminated immunologic response to exogenous antigen. $J$ Immuno/85,403-17(1960)

[26] Shevach EM. Foxp3(+) T Regulatory Cells: Still Many Unanswered Questions-A Perspective After 20 Years of Study. Frontiers in immunology. 9,1048(2018)

[27] Fontenot JD, Gavin MA, Rudensky AY. Foxp3 programs the development and function of CD4+CD25+ regulatory T cells. Nature immunology.4,330-6(2003)

[28] Bennett CL, Christie J, Ramsdell F, Brunkow ME, Ferguson PJ, Whitesell L, et al. The immune dysregulation, polyendocrinopathy, enteropathy, X-linked syndrome (IPEX) is caused by mutations of FOXP3. Nature genetics. 27,20-1(2001)

[29] Brunkow ME, Jeffery EW, Hjerrild KA, Paeper B, Clark LB, Yasayko SA, et al. Disruption of a new forkhead/winged-helix protein, scurfin, results in the fatal lymphoproliferative disorder of the scurfy mouse. Nature genetics.,27,68-73(2001)

[30] Hori S, Nomura T, Sakaguchi S. Control of regulatory T cell development by the transcription factor Foxp3. Science (New York, NY).299,1057-61(2003)

[31] Liu W, Putnam AL, Xu-yu Z, Szot GL, Lee MR, Zhu S, et al. CD127 expression inversely correlates with FoxP3 and suppressive function of human CD4+ T reg cells. Journal of Experimental Medicine. 203,170111(2006)

[32] Wang C, Yu X, Yan Y, Yang W, Zhang S, Xiang Y, et al. Tumor necrosis factor-a: a key contributor to intervertebral disc degeneration. Acta biochimica et biophysica Sinica. 49,1-13(2017) 
[33] Saha P, Smith A. TNF-a (Tumor Necrosis Factor-a). Arteriosclerosis, thrombosis, and vascular biology.38,2542-3(2018)

[34] Gaffen SL, Liu KD. Overview of interleukin-2 function, production and clinical applications. Cytokine.28,109-23(2004)

[35] Malek TR, Yu A, Vincek V, Scibelli P, Kong L. CD4 regulatory T cells prevent lethal autoimmunity in IL2Rbeta-deficient mice. Implications for the nonredundant function of IL-2. Immunity.17,167-78(2002)

[36] Malek TR, Porter BO, Codias EK, Scibelli P, Yu A. Normal lymphoid homeostasis and lack of lethal autoimmunity in mice containing mature T cells with severely impaired IL-2 receptors. Journal of immunology (Baltimore, Md : 1950). 164,2905-14(2000)

[37] Tian G, Li JL, Wang DG, Zhou D. Targeting IL-10 in auto-immune diseases. Cell biochemistry and biophysics. 70,37-49(2014)

\section{Figures}
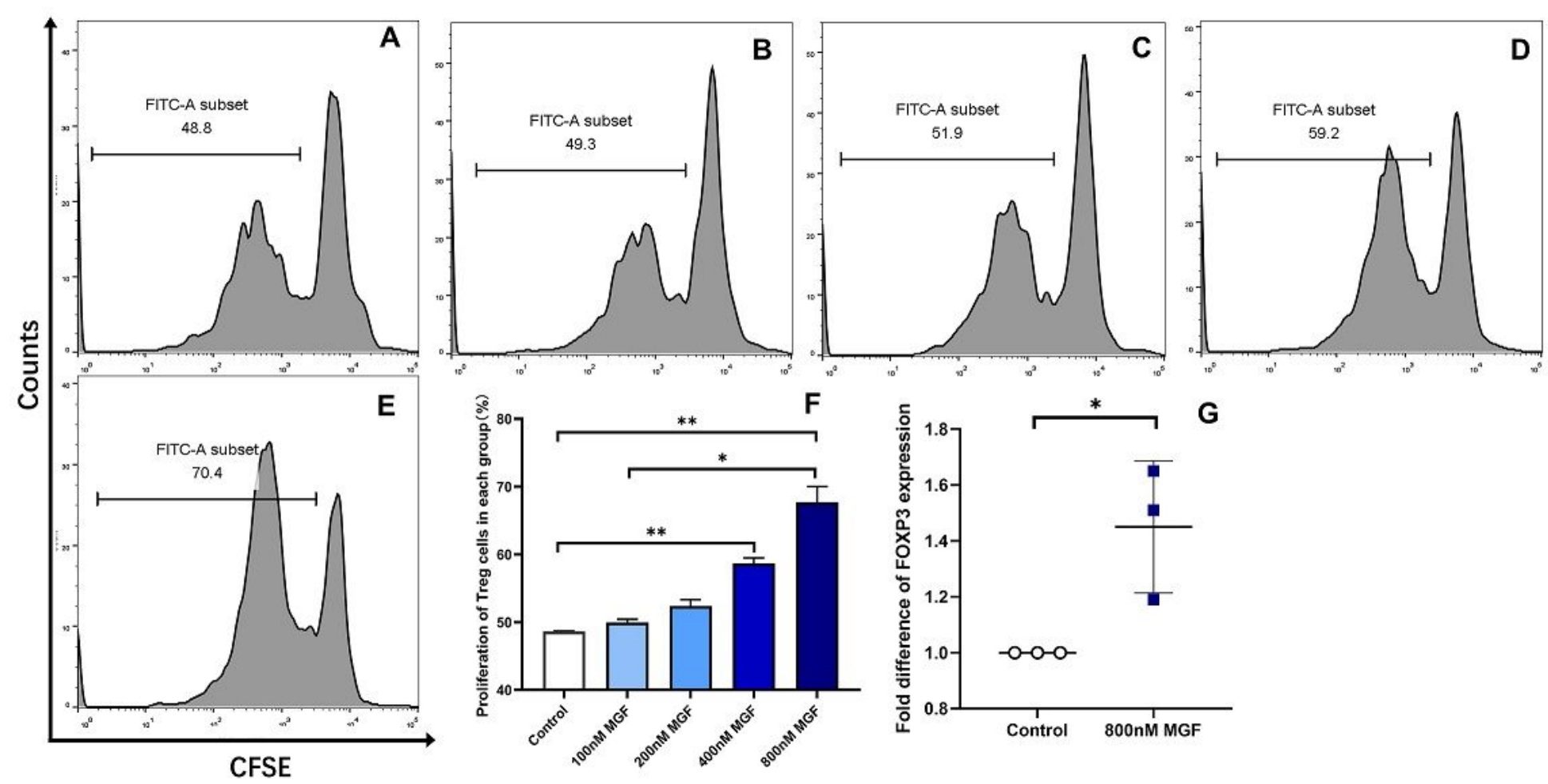

Figure 1

Proliferation of Treg cells in each group and fold-change in the expression levels of FOXP3 in Treg cells. Average percentage of Treg cells in the (A) control group, (B) $100 \mathrm{~nm}$ MGF group, (C) $200 \mathrm{~nm}$ MGF group, (D) 400 nm MGF group, and (E) 800 nm MGF group. (F) Proliferation of Treg cells in each group. (G) Foldchange in the expression levels of FOXP3 in Treg cells in three experiments. 


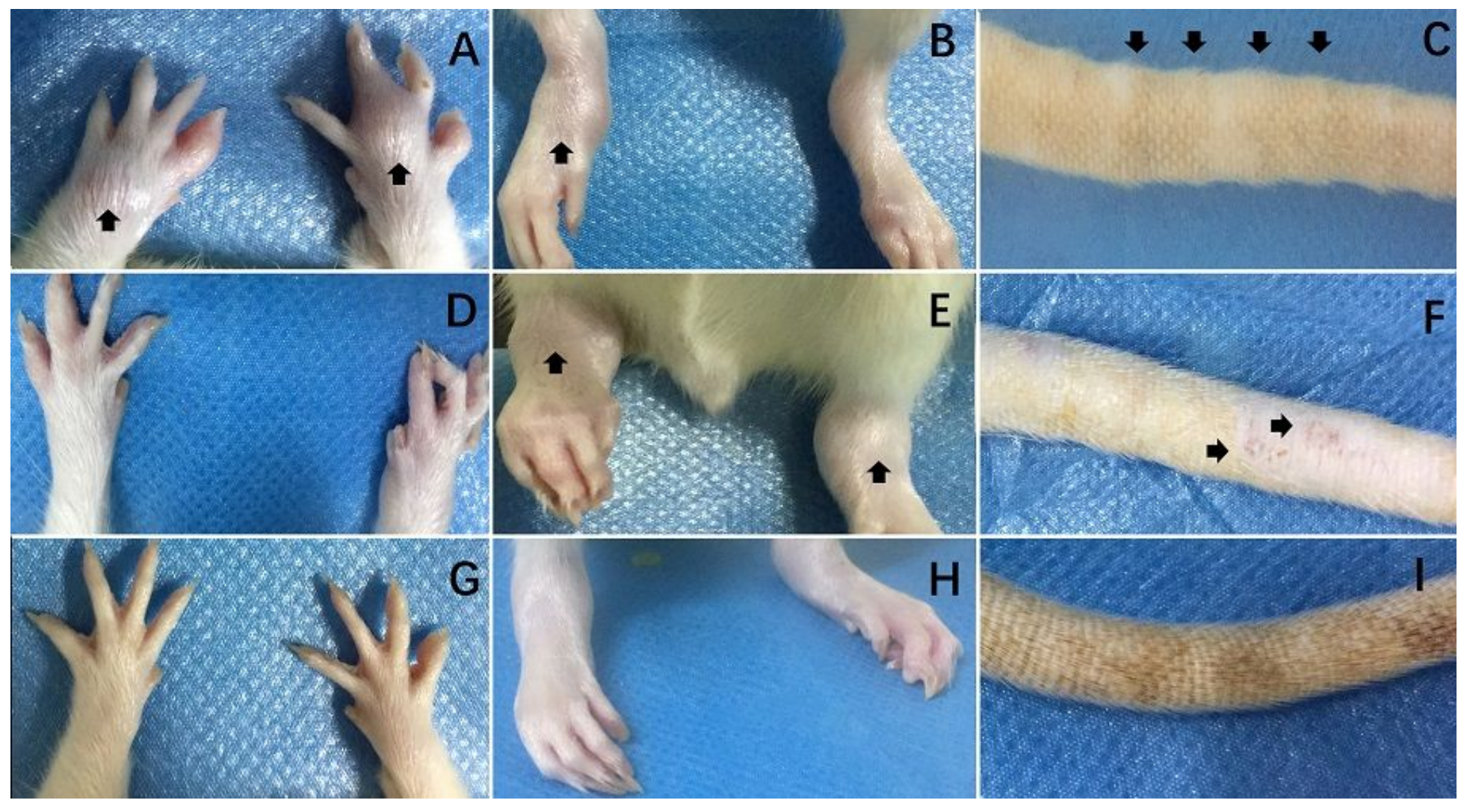

Figure 2

Clinical manifestations of HLA-B27/Huß2m rats in each group. (A-C) Control group. (D-F) Low-dose MGF group. (G-I) High-dose MGF group. Up arrows indicate swollen joints. Down arrows indicate bamboo-like change. Right arrows indicate skin erythema. 

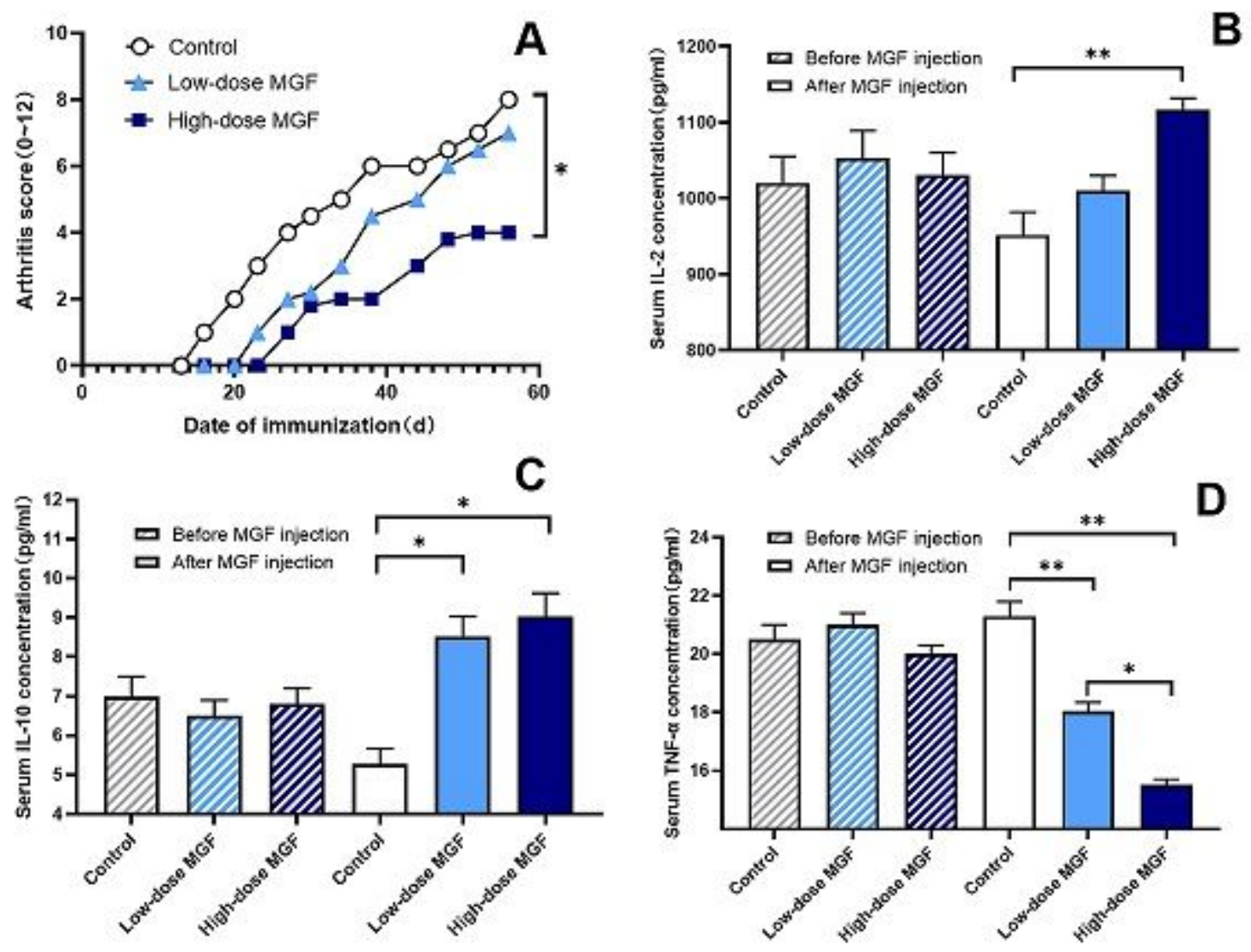

\section{Figure 3}

Arthritis clinical scores in each group and the comparison of serum IL-2, IL-10, and TNF-a levels before and after MGF treatment in HLA-B27/Huß2m rats. (A) HLA-B27/Huß2m rat arthritis clinical scores in each group. (B) Serum IL-2 level before and after MGF treatment in HLA-B27/Huß2m rats. (C) Serum IL-10 level before and after MGF treatment in HLA-B27/Huß2m rats. (D) Serum TNF-a level before and after MGF treatment in HLAB27/Huß2m rats. 


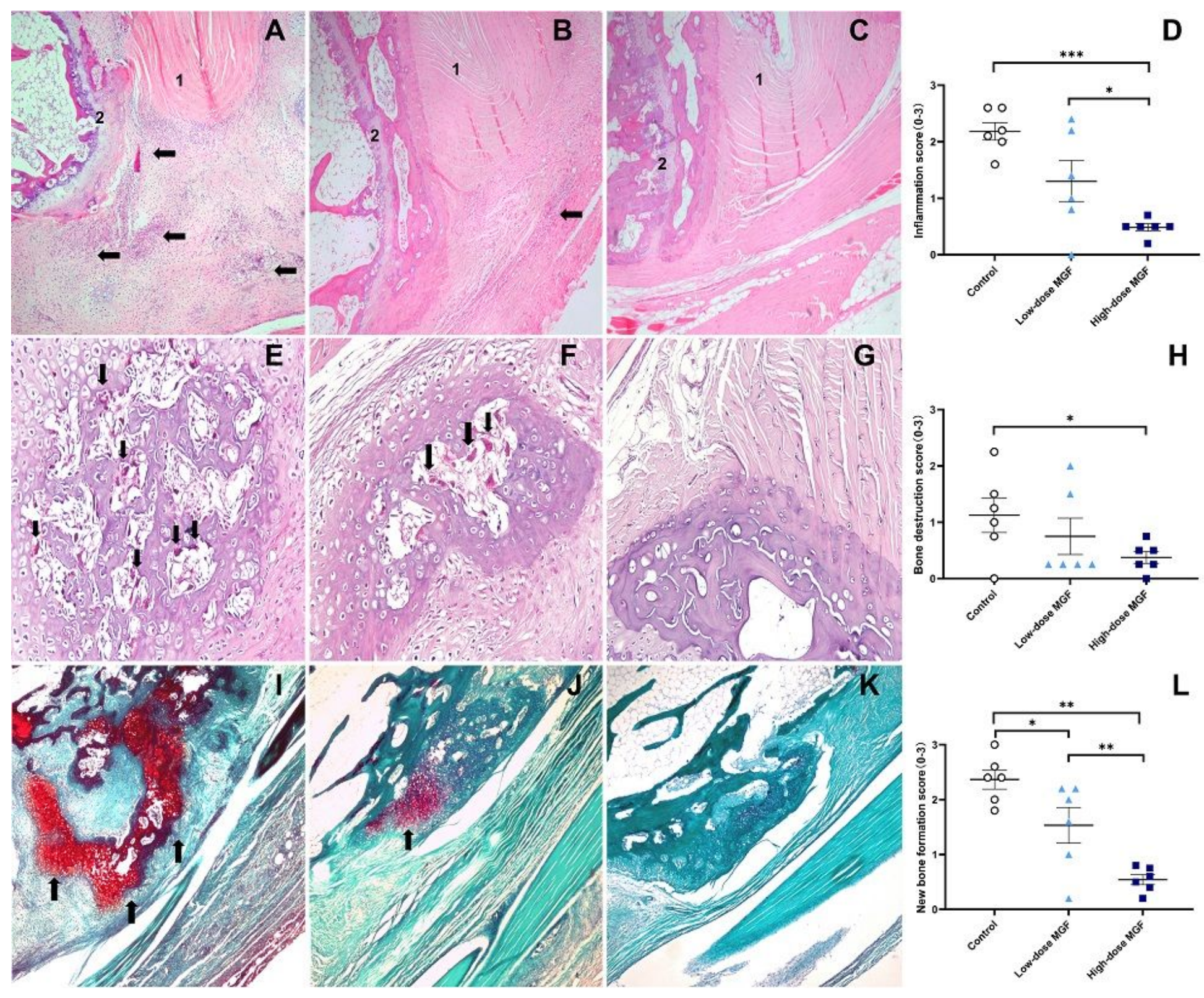

Figure 4

H\&E staining and safranin 0 staining scoring of HLA-B27/Huß2m rat tissue in each group. (A-C) Representative H\&E staining of joint inflammation in the (A) control group, (B) low-dose MGF group, and (C) high-dose MGF group. (D) Inflammation infiltration score. From A to $C$, the number of inflammatory cells and range of infiltration gradually decreased. "1" indicates annulus fibrosus, "2" indicates spine bone, and black arrows indicate inflammatory cell aggregation. Left arrow indicates inflammatory cell infiltration. (E-H) H\&E staining of joint bone destruction in the (E) control group, (F) low-dose MGF group, and $(\mathrm{G})$ high-dose MGF group. $(\mathrm{H})$ Bone-destruction score. From $E$ to $G$, the degree of bone destruction gradually decreased, and the number of multinucleated osteoclasts gradually decreased. Up arrows indicate multinucleated osteoclasts. (I-K) Safranin $\mathrm{O}$ staining of new joint bone formation in the (I) control group, (J) low-dose MGF group, and (K) high-dose MGF group. (L) New bone formation score. From I to $\mathrm{K}$, the area of new cartilage gradually decreased. Down arrows indicate new cartilage tissue. 


\section{Supplementary Files}

This is a list of supplementary files associated with this preprint. Click to download.

- supplementaldata.docx

- supplementaldataFig1.jpg 